\title{
Prevalence of Clostridioides difficile and Other Gastrointestinal Pathogens in Patients with COVID-19
}

\author{
Monika Laszkowska ${ }^{1,2}\left(\right.$ ) Judith Kim ${ }^{1} \cdot$ Adam S. Faye $^{1,3} \cdot$ Andrew M. Joelson $^{1} \cdot$ Myles Ingram $^{4} \cdot$ Han Truong $^{4}$. \\ Elisabeth R. Silver ${ }^{4} \cdot$ Benjamin May $^{5}$. William G. Greendyke ${ }^{6}$. Jason Zucker ${ }^{6}$. Benjamin Lebwohl ${ }^{1,7}$. Chin Hur ${ }^{1,4,5}$. \\ Daniel E. Freedberg ${ }^{1}$
}

Received: 29 August 2020 / Accepted: 4 December 2020 / Published online: 22 January 2021

(c) The Author(s), under exclusive licence to Springer Science+Business Media, LLC part of Springer Nature 2021

\begin{abstract}
Background Gastrointestinal symptoms are common in patients with COVID-19, but prevalence of co-infection with enteric pathogens is unknown.

Aims This study assessed the prevalence of enteric infections among hospitalized patients with COVID-19.

Methods We evaluated 4973 hospitalized patients $\geq 18$ years of age tested for COVID-19 from March 11 through April 28, 2020, at two academic hospitals. The primary exposure was a positive COVID-19 test. The primary outcome was detection of a gastrointestinal pathogen by PCR stool testing.

Results Among 4973 hospitalized individuals, 311 were tested for gastrointestinal infections (204 COVID-19 positive, 107 COVID-19 negative). Patients with COVID-19 were less likely to test positive compared to patients without COVID-19 $(10 \%$ vs $22 \%, p<0.01)$. This trend was driven by lower rates of non-C.difficile infections ( $11 \%$ vs $22 \%$ in COVID-19 positive vs. negative, respectively, $p=0.04)$, but not $C$. difficile infection $(5.1 \%$ vs. $8.2 \%, p=0.33)$. On multivariable analysis, infection with COVID-19 remained significantly associated with lower odds of concurrent GI infection (aOR 0.49, 95\% CI 0.24-0.97), again driven by reduced non-C.difficile infection. Testing for both C.difficile and non-C.difficile enteric infection decreased dramatically during the pandemic.

Conclusions Pathogens aside from C.difficile do not appear to be a significant contributor to diarrhea in COVID-19 positive patients.
\end{abstract}

Keywords COVID-19 $\cdot$ SARS-CoV-2 $\cdot$ Clostridioides difficile $\cdot$ Enteric infection $\cdot$ Diarrhea

\author{
Abbreviations \\ C. difficile Clostridioides difficile \\ GI Gastrointestinal
}

COVID-19 Coronavirus Disease 2019

SARS-CoV-2 Severe Acute Respiratory Syndrome Coronavirus 2

Supplementary information The online version contains supplementary material available at https://doi.org/10.1007/s1062 0-020-06760-y.

Monika Laszkowska

m13228@caa.columbia.edu

1 Department of Medicine, Division of Digestive and Liver Diseases, New York Presbyterian Columbia University Medical Center, New York, NY, USA

2 Department of Medicine, Gastroenterology, Hepatology, and Nutrition Service, Memorial Sloan Kettering Cancer Center, New York, NY, USA

3 Department of Medicine, Henry D. Janowitz Division of Gastroenterology, Mount Sinai Hospital, New York, NY, USA
4 Department of Medicine, Division of General Medicine, Columbia University Irving Medical Center, New York, NY, USA

5 Herbert Irving Comprehensive Cancer Center, Columbia University Irving Medical Center, New York, NY, USA

6 Department of Medicine, Division of Infectious Diseases, Columbia University Irving Medical Center, New York, NY, USA

7 Department of Epidemiology, Mailman School of Public Health, Columbia University Irving Medical Center, New York, NY, USA 


\section{Introduction}

The 2019 coronavirus disease (COVID-19) has become a global pandemic with cases spreading rapidly throughout the world. While fever and cough are the most common symptoms associated with this disease, gastrointestinal manifestations have also been noted in 32 to $61 \%$ of individuals [1-8]. Diarrhea, in particular, has been reported in 4 to $34 \%$ of individuals in cohorts from China and 12 to $34 \%$ in cohorts from the USA [2-8].

A likely mechanism for the prevalence of gastrointestinal symptoms in patients with COVID-19 is the presence of Angiotensin Converting Enzyme 2 (ACE2) receptors on gut epithelial cells, a well-described target for viral binding and entry $[9,10]$. Nonetheless, it is also possible that intestinal inflammation due to COVID-19 could predispose individuals to co-infection with other gastrointestinal pathogens. Thus far, only one prior study from Wuhan, China, evaluated whether co-infection with enteric pathogens plays a prominent role in this presentation, finding no evidence of enteric pathogens by stool culture in 74 patients with COVID-19 and gastrointestinal symptoms [8].

Highly sensitive and specific stool polymerase chain reaction-based assays have improved the ability to diagnose gastrointestinal infections, including those caused by Clostridioides difficile ( $C$. difficile), and are routinely used at our institution $[11,12]$. Such infections, particularly C.difficile, can cause a significant burden to infected individuals thus making recognition and treatment key $[13,14]$. This retrospective cohort study aimed to evaluate the prevalence of co-infection with gastrointestinal pathogens among admitted patients with and without COVID-19 infection. In addition, we aimed to compare institution-wide patterns in gastrointestinal infection testing and rates of positive cases before and after the start of the COVID-19 pandemic in New York City.

\section{Methods}

\section{Study Design}

This retrospective study evaluated 4973 hospitalized patients $\geq 18$ years of age who underwent testing for COVID-19 from March 11, 2020, (when institution-based testing began) through April 28, 2020, at two affiliated academic medical centers (Columbia University Irving Medical Center and Allen Hospital). The primary exposure of interest was a positive COVID-19 test, defined as a positive result on real-time reverse-transcriptase-polymerase-chainreaction (RT-PCR) assay of nasopharyngeal swab specimens testing for the presence of severe acute respiratory syndrome coronavirus 2 (SARS-CoV-2). The primary outcome of interest was the detection of a gastrointestinal (GI) pathogen $(C$. difficile and non- $C$. difficile) during the index hospitalization by PCR test of the stool. Data regarding duration of GI symptoms with COVID-19 is limited, but one study from China found that COVID-19 positive patients with GI symptoms alone had on average 16 days from initial symptom onset to hospital admission and 31 days from symptom onset to viral clearance [15]. Therefore, stool tests performed either 2 weeks before or at any time after SARS-CoV-2 testing were included in order to capture an adequate time period during which patients may have had symptoms related to COVID-19. The institutional laboratory policy during this period was that only unformed stools would be tested for enteric pathogens (i.e., implying that all tested patients did indeed have diarrhea). It was at the discretion of the provider caring for the patient whether to order a C.difficile PCR stool test, a gastrointestinal pathogen PCR panel stool test, or both tests. Secondary variables of interest were age, sex, body mass index (BMI), and race/ethnicity. Stool studies performed from February 1, 2020 to April 17, 2020 were used to determine trends in testing patterns before and during the pandemic.

\section{Gastrointestinal Pathogen PCR Panel Stool Test}

Gastrointestinal pathogens were identified using the FilmArray gastrointestinal pathogen PCR panel (GI PCR; BioFire Diagnostics, Salt Lake City, UT). This assay allows for simultaneous detection and identification of nucleic acids from multiple bacteria, viruses, and parasites directly from spontaneously voided stool samples. The assay identifies 20 pathogens including Adenovirus, Astrovirus, Campylobacter, Cryptosporidium, Cyclospora, Entamoeba histolytica, Enteroaggregative E. coli, Enteropathogenic E. coli, Enterotoxigenic E. coli, Giardia, Norovirus, Plesiomonas shigelloides, Rotavirus, Salmonella species, Sapovirus, Shigalike toxin-producing E. coli STX/ST2, Shigella/Enteroinvasive E. coli, Vibrio cholerae, Vibrio species, and Yersinia enterocolitica.

\section{Clostridioides difficile Testing}

C.difficile PCR testing was done via PCR for the toxin B gene (Xpert C. difficile, Cepheid, Sunnyvale, CA). At our institution, this test is ordered separately from and/or in addition to the GI PCR test panel at the discretion of the ordering provider. There is an institutional electronic "hard stop" that prohibits stool PCR testing in patients who have received laxatives within the previous $24 \mathrm{~h}$. 


\section{Statistical Analysis}

Continuous variables were expressed as medians or interquartile ranges, with Wilcoxon rank-sum test used to assess differences in distributions between the groups. Categorical variables were summarized as counts and percentages, with Chi-square or Fisher's exact tests used for comparison. A multivariable model was built to examine whether COVID-19 was independently associated with gastrointestinal infection after adjusting for all included variables. A modified Charlson comorbidity index was utilized, which incorporated weighted values of all components of the index (myocardial infarction, congestive heart failure, peripheral vascular disease, cerebrovascular disease, dementia, chronic obstructive pulmonary disease, connective tissue disease, peptic ulcer disease, diabetes mellitus, chronic kidney disease, hemiplegia, leukemia, lymphoma, solid tumor, liver disease, and AIDS) but excluded age since it was included separately in our multivariable model. An alpha of 0.05 was considered statistically significant. Statistical calculations were performed in STATA 16 (Stata Corp, College Station, TX). This study was approved by the Institutional Review Board of Columbia University Medical Center.

\section{Results}

We identified 4973 individuals requiring inpatient admission who were tested for COVID-19 between March 11, 2020 and April 28, 2020 at two affiliated academic centers in New York City. 2870 (58\%) were COVID-19 positive and 2103 (42\%) tested negative. Among these individuals, 311 were also tested for gastrointestinal infections in either the 2 weeks prior or at any time after their SARS-CoV-2 PCR, including 204 COVID-19 positive and 107 COVID19 negative patients. 261 patients were tested for $C$. difficile infection and 195 patients were tested for non- $C$. difficile infection, with 145 patients being tested for both.

Of the 311 patients with stool studies, there was no difference in age or sex between COVID-19 positive and negative individuals (see Table 1). Significantly more non-Hispanic whites were tested in the COVID-19 negative group (31\%, compared to $16 \%$ in positive patients), while more Hispanics were tested among the COVID-19 positive cohort (35\%, compared to $30 \%$ in negatives; see Table 1). More of the COVID-19 positive patients were also overweight or obese with a BMI $\geq 25$ (69\% vs. $48 \%, p<0.01)$. There was a significantly higher proportion of COVID-19 negative patients with a modified Charlson comorbidity index $\geq 2$ than COVID-19 positive patients (68\% vs. $34 \%$ respectively, $p<0.01$ ).

COVID-19 positive patients had a significantly longer median length of stay than COVID negative patients (14.6 vs. 7.7 , respectively, $p<0.01)$ and were more likely to be tested for $C$. difficile later during hospitalization (73\% tested $>72 \mathrm{~h}$ after admission, compared to $52 \%$ in COVID19 negative patients; $p<0.01$; see Table 1 ). However, there was minimal difference in the timing of testing for non- $C$. difficile enteric pathogens, comparing those with versus those without COVID-19 ( $p=0.06$, Table 1). Among all 4,973 patients, COVID-19 positive patients were tested more often for $C$. difficile compared to COVID-19 negative patients, but there was no significant difference in rates of testing for other GI pathogens (Supplementary Table 1).

COVID-19 positive patients were less likely to have any GI infection overall compared to COVID-19 negative patients ( $10 \%$ vs $22 \%, p<0.01$, Table 2$)$. This trend persisted when evaluating only non- $C$. difficile infection $(11 \%$ vs $22 \%, p=0.04)$. However, no significant difference was seen between the two groups in testing positive for $C$. diffcile infection. Of the non-C. difficile pathogens, enteropathogenic $E$. coli and enteroaggregative $E$. coli were the most common, found in $2.7 \%$ and $2.9 \%$ of patients with a GI PCR test, respectively. Seven patients had multiple GI pathogens detected on the GI PCR panel, including five patients with two pathogens detected and two patients with three pathogens detected. Of these, four were COVID-19 positive and three were negative. One additional patient had $C$. difficile infection as well as a second pathogen detected on the GI PCR panel. There were no significant differences in the rates or types of gastrointestinal pathogens detected in individuals with severe COVID-19 (resulting in intubation and/or death) and those with a milder course of COVID-19 (Supplementary Tables 2 and 3 ).

Of individuals with stool studies during the study period, those with COVID-19 were less likely to have a GI infection on univariable analysis ( $69 \%$ vs $47 \%, p<0.01$, Table 3$)$. On multivariable analysis, infection with COVID-19 remained significantly associated with lower odds of GI infection in tested patients after adjusting for age, sex, BMI, and race/ ethnicity (aOR 0.49, 95\% CI 0.24-0.97, $p=0.04$, Table 4).

We also evaluated the trend in GI PCR and $C$. difficile testing before and after the beginning of the COVID-19 pandemic. COVID-19 testing became widely available at our institution on March 11, 2020. As shown in Fig. 1, as of March 14, testing for both $C$. difficile and non-C. difficile enteric infection decreased dramatically, with the lowest volume of testing observed at the end of March. In April, $C$. difficile testing increased, approaching pre-pandemic rates, whereas GI PCR testing rates remained lower than pre-pandemic rates. 
Table 1 Characteristics of COVID-19+ and COVID-19 patients with stool studies

\begin{tabular}{|c|c|c|c|c|}
\hline Variables & $\begin{array}{l}\text { All } \\
n=311\end{array}$ & $\begin{array}{l}\text { COVID }+n(\%) \\
n=204\end{array}$ & $\begin{array}{l}\text { COVID- } n(\%) \\
n=107\end{array}$ & $p$ Value \\
\hline \multicolumn{5}{|l|}{ Age, years } \\
\hline Mean (SD) & $62.5(16.5)$ & $62.9(15.6)$ & $61.7(19.3)$ & 0.57 \\
\hline Median (IQR) & $64.7(53.2-74.6)$ & $63.9(53.6-73.2)$ & $65.9(50.3-75.5)$ & 0.98 \\
\hline \multicolumn{5}{|l|}{ Distribution $n(\%)$} \\
\hline $18-39$ & $36(12)$ & $20(10)$ & $16(15)$ & 0.25 \\
\hline $40-59$ & $75(24)$ & $51(25)$ & $24(22)$ & \\
\hline $60-74$ & $125(40)$ & $88(43)$ & $37(35)$ & \\
\hline $75+$ & $75(24)$ & $45(22)$ & $30(28)$ & \\
\hline Male Sex $n(\%)$ & $187(60)$ & $125(61)$ & $62(58)$ & 0.57 \\
\hline \multicolumn{5}{|l|}{ Race/ethnicity $n(\%)$} \\
\hline Non-Hispanic White & $66(21)$ & $33(16)$ & $33(31)$ & 0.01 \\
\hline Hispanic & $103(33)$ & $71(35)$ & $32(30)$ & \\
\hline Black & $80(26)$ & $51(25)$ & $29(27)$ & \\
\hline Asian & $5(2)$ & $4(2)$ & $1(1)$ & \\
\hline Other & $57(18)$ & $45(22)$ & $12(11)$ & \\
\hline \multicolumn{5}{|l|}{$\mathrm{BMI} n(\%)$} \\
\hline$<25$ & $95(30)$ & $50(25)$ & $45(42)$ & $<0.01$ \\
\hline $25-30$ & $90(30)$ & $67(33)$ & $23(22)$ & \\
\hline$>30$ & $102(33)$ & $74(36)$ & $28(26)$ & \\
\hline Unknown & $24(7)$ & $13(6)$ & $11(10)$ & \\
\hline \multicolumn{5}{|l|}{ Modified Charlson comorbidity } \\
\hline \multicolumn{5}{|l|}{ Score } \\
\hline 0 & $90(29)$ & $68(33)$ & $22(21)$ & $<0.01$ \\
\hline 1 & $59(19)$ & $47(23)$ & $12(11)$ & \\
\hline $2+$ & $162(52)$ & $89(34)$ & $73(68)$ & \\
\hline \multicolumn{5}{|c|}{ Time of stool studies relative to SARS-CoV-2 test } \\
\hline \multicolumn{5}{|l|}{ C. difficile $(n=261)$} \\
\hline 2 weeks before & $13(5)$ & $7(4)$ & $6(7)$ & 0.51 \\
\hline 30 days after & $239(92)$ & $157(92)$ & $82(91)$ & \\
\hline$>30$ days after & $9(3)$ & $7(4)$ & $2(2)$ & \\
\hline \multicolumn{5}{|l|}{ Other GI pathogens $(n=195)$} \\
\hline 2 weeks before & $15(8)$ & $5(4)$ & $10(13)$ & 0.03 \\
\hline 30 days after & $180(92)$ & $112(96)$ & $68(87)$ & \\
\hline$>30$ days after & $0(0)$ & - & - & \\
\hline \multicolumn{5}{|c|}{ Time of stool studies relative to Admission } \\
\hline \multicolumn{5}{|l|}{ C. difficile } \\
\hline Before admission & $7(3)$ & $3(2)$ & $4(5)$ & $<0.01$ \\
\hline Within $72 \mathrm{~h}$ of admission & $82(31)$ & $45(25)$ & $37(43)$ & \\
\hline$>72 \mathrm{~h}$ post-admission & $172(66)$ & $128(73)$ & $44(52)$ & \\
\hline \multicolumn{5}{|l|}{ Other GI pathogens } \\
\hline Before admission & $7(4)$ & $2(2)$ & $5(6)$ & 0.06 \\
\hline Within $72 \mathrm{~h}$ of admission & $94(48)$ & $52(44)$ & $42(54)$ & \\
\hline$>72 \mathrm{~h}$ post-admission & $94(48)$ & $63(54)$ & $31(40)$ & \\
\hline Length of stay ${ }^{\dagger}-$ Median (IQR) & $12.5(6.2-25.1)$ & $14.6(8.3-26.3)$ & $7.7(4.7-17.1)$ & $<0.01$ \\
\hline
\end{tabular}

${ }^{\dagger}$ Patients still currently admitted were censored at the conclusion of the study on 4/28/20 
Table 2 GI pathogen detection rates in COVID-19+ and COVID-19-patients with stool studies

\begin{tabular}{|c|c|c|c|c|}
\hline Variables & All & $\mathrm{COVID}+n(\%)$ & COVID- $n(\%)$ & $P$ value \\
\hline \multicolumn{5}{|l|}{ Any GI pathogen, $n=311$} \\
\hline Positive & $45(14.5)$ & $21(10.3)$ & $24(22.4)$ & \multirow[t]{2}{*}{$<0.01^{*}$} \\
\hline Negative & $266(85.5)$ & $183(89.7)$ & $83(77.6)$ & \\
\hline \multicolumn{5}{|l|}{ C. difficile, $n=261$} \\
\hline Positive & $16(6.1)$ & $9(5.1)$ & $7(8.2)$ & \multirow[t]{2}{*}{0.33} \\
\hline Negative & 245 (93.9) & $167(94.9)$ & $78(91.8)$ & \\
\hline \multicolumn{5}{|l|}{ Other GI pathogens, $n=195$} \\
\hline Positive & $30(15.4)$ & $13(11.1)$ & $17(21.8)$ & \multirow[t]{2}{*}{$0.04 *$} \\
\hline Negative & $165(84.6)$ & $104(88.9)$ & $61(78.2)$ & \\
\hline \multicolumn{5}{|l|}{ Other GI pathogens, $n=195$} \\
\hline Adenovirus & $0(0)$ & $0(0)$ & $0(0)$ & \\
\hline Astrovirus & $0(0)$ & $0(0)$ & $0(0)$ & \\
\hline Campylobacter & $2(0.6)$ & $1(0.5)$ & $1(0.9)$ & \\
\hline Cryptosporidium & $1(0.3)$ & $1(0.5)$ & $0(0)$ & \\
\hline Cyclospora & $0(0)$ & $0(0)$ & $0(0)$ & \\
\hline Entameoba histolytica & $0(0)$ & $0(0)$ & $0(0)$ & \\
\hline Enteroaggregative E. coli & $8(2.7)$ & $4(2.0)$ & $4(3.7)$ & \\
\hline Enteropathogenic E. coli & $9(2.9)$ & $5(2.5)$ & $4(3.7)$ & \\
\hline Enterotoxigenic $E$. coli & $0(0)$ & $0(0)$ & $0(0)$ & \\
\hline Giardia & $1(0.3)$ & $1(0.5)$ & $0(0)$ & \\
\hline Norovirus & $4(1.3)$ & $1(0.5)$ & $3(2.8)$ & \\
\hline Plesiomonas shigelloides & $0(0)$ & $0(0)$ & $0(0)$ & \\
\hline Rotavirus & $4(1.3)$ & $1(0.5)$ & $3(2.8)$ & \\
\hline Salmonella species & $2(0.6)$ & $2(1.0)$ & $0(0)$ & \\
\hline Sapovirus & $2(0.6)$ & $0(0)$ & $2(1.9)$ & \\
\hline $\begin{array}{l}\text { Shigalike toxin-producing } E \text {. } \\
\text { coli STX/ST2 }\end{array}$ & $2(0.6)$ & $1(0.5)$ & $1(0.9)$ & \\
\hline Shigella/enteroinvasive E. coli & $2(0.6)$ & $0(0)$ & $2(1.9)$ & \\
\hline Vibrio cholerae & $0(0)$ & $0(0)$ & $0(0)$ & \\
\hline Vibrio species & $0(0)$ & $0(0)$ & $0(0)$ & \\
\hline Yersinia enterocolitica & $0(0)$ & $0(0)$ & $0(0)$ & \\
\hline
\end{tabular}

\section{Discussion}

In this retrospective study of hospitalized patients tested for COVID-19, we found that patients with COVID-19 were less likely to test positive for co-infection with typical gastrointestinal pathogens compared to those without COVID-19. This difference was driven by non- $C$. difficile pathogens. $C$. difficile infection rates were similar among COVID-19 positive and negative patients, although patients with COVID-19 had longer median hospital stays and were diagnosed with $C$. difficile later during the course of hospitalization.

Despite the high prevalence of gastrointestinal symptoms described in COVID-19 patients [3-8], a relatively small percentage of patients overall were tested during the study period. Overall, rates of $C$. difficile testing were relatively unchanged by the COVID-19 pandemic whereas rates of testing for other GI pathogens were less than half normal.
Our study suggests that $C$. difficile should remain in the differential for patients presenting with diarrhea regardless of COVID-19 status, especially later in their admission, given that patients with COVID-19 had $C$. difficile infections at the same rates as those without COVID-19. Other gastrointestinal pathogens may be less likely in patients with COVID19 , though this may have been affected by decreased rates of testing.

To our knowledge, this is the first study to report the prevalence of co-infection with enteric pathogens using PCR-based stool assays in patients under evaluation for COVID-19. Disruption of normal mucosal defenses by one enteric infection is recognized as a risk factor for other infections, and prior studies have found that up to $26 \%$ of patients undergoing stool pathogen testing have multiple pathogens detected [16]. Only $0.9 \%$ of the overall study population were positive for an enteric infection, 
Table 3 Univariable predictors of GI pathogen detection in patients with stool studies

\begin{tabular}{|c|c|c|c|c|}
\hline Variables & All tested $n=311$ & $\begin{array}{l}\text { GI pathogen } \\
\text { detected } n=45\end{array}$ & $\begin{array}{l}\text { No GI pathogen } \\
\text { detected } \\
n=266\end{array}$ & $p$ Value \\
\hline COVID-19 infection & $204(66)$ & $21(47)$ & $183(69)$ & $<0.01$ \\
\hline Age, years mean (SD) & $62.0(16.5)$ & $65.5(14.8)$ & $62.0(16.8)$ & 0.91 \\
\hline Male sex $n(\%)$ & $187(60)$ & $29(64)$ & $158(59)$ & 0.52 \\
\hline \multicolumn{5}{|l|}{ Racelethnicity n (\%) } \\
\hline Non-Hispanic white & $66(21)$ & $13(20)$ & $53(20)$ & 0.25 \\
\hline Hispanic & $103(33)$ & $17(37)$ & $86(32)$ & \\
\hline Black & $80(26)$ & $10(22)$ & $70(26)$ & \\
\hline Other & $62(20)$ & $5(11)$ & $57(21)$ & \\
\hline \multicolumn{5}{|l|}{$B M I n(\%)$} \\
\hline$<25 \mathrm{~kg} / \mathrm{m} 2$ & $95(30)$ & $17(38)$ & $78(29)$ & 0.08 \\
\hline $25-30 \mathrm{~kg} / \mathrm{m} 2$ & $90(29)$ & $14(31)$ & $76(29)$ & \\
\hline$>30 \mathrm{~kg} / \mathrm{m} 2$ & $102(33)$ & $8(18)$ & $94(35)$ & \\
\hline Unknown & $24(8)$ & $6(13)$ & $18(7)$ & \\
\hline \multicolumn{5}{|c|}{ Modified Charlson comorbidity score } \\
\hline 0 points & $90(28.9)$ & $8(17.8)$ & $82(30.8)$ & 0.09 \\
\hline 1 point & $59(19.0)$ & $7(15.6)$ & $52(19.6)$ & \\
\hline $2+$ points & $162(52.1)$ & $30(66.6)$ & $132(49.6)$ & \\
\hline
\end{tabular}

Table 4 Multivariable model of predictors of GI pathogen detection in tested patients $(n=311)$

\begin{tabular}{llll}
\hline Variables & Odds ratio & Confidence interval & $p$ Value \\
\hline COVID-19 infection & 0.49 & $0.24-0.97$ & 0.04 \\
Age (per additional year) & 1.01 & $0.99-1.03$ & 0.38 \\
Male sex & 1.31 & $0.66-2.62$ & 0.44 \\
Race/ethnicity & & & \\
Non-Hispanic white & Reference & - & \\
Hispanic & 0.98 & $0.43-2.27$ & 0.97 \\
Black & 0.7 & $0.28-1.77$ & 0.45 \\
Other & 0.42 & $0.13-1.31$ & 0.14 \\
BMI & & & \\
$<25 \mathrm{~kg} / \mathrm{m} 2$ & Reference & - & \\
$25-30 \mathrm{~kg} / \mathrm{m} 2$ & 1.1 & $0.48-2.51$ & 0.81 \\
$>30 \mathrm{~kg} / \mathrm{m} 2$ & 0.5 & $0.20-1.34$ & 0.18 \\
Unknown & 1.44 & $0.48-4.38$ & 0.51 \\
Modified Charlson comorbidity score & & \\
0 points & Reference & - & \\
1 point & 1.65 & $0.54-5.00$ & 0.38 \\
$2+$ points & 2.04 & $0.85-4.89$ & 0.11 \\
\hline
\end{tabular}

although the prevalence of infections among those who were actually tested for enteric pathogens was much higher, at $14.5 \%$. The majority of gastrointestinal symptoms in COVID-19 patients may be attributable to the virus itself. This is in line with the findings of the study by Jin, et al. where 0 of 74 COVID-19 patients in China with gastrointestinal symptoms had positive stool cultures [8]. Aside from $C$. difficile, the most commonly identified enteric infections were $E$. coli species, which would be expected given these are relatively common pathogens. Although a similar number of COVID-19 positive and COVID-19 negative patients had multiple pathogens detected on GI PCR, this was observed in a small number of patients making it difficult to draw definitive conclusions regarding risks of co-infection in these populations.

Patients with COVID-19 likely have low rates of non- $C$. difficile enteric infections because SARS-CoV-2 itself is the cause of diarrhea on presentation; however, over time these patients become more similar to other hospitalized patients and have a similar rate of $C$. difficile infections compared to patients without COVID-19. This is particularly notable given that the standard of care for COVID-19 positive patients includes heightened contact precautions and hand hygiene. Furthermore, while COVID-19 positive patients had fewer comorbidities than COVID-19 negative patients, the similar rates of $C$. difficile infections in these groups speak to the power of prolonged hospitalization as a risk factor for this infection.

We also found that testing for enteric infections decreased at our institution since the onset of the COVID-19 pandemic. There are several possible explanations for this. First, during the COVID-19 pandemic, patients may be less likely to seek care for symptoms that may otherwise prompt presentation to a hospital, such as diarrhea. Second, some providers evaluating patients with diarrhea may attribute these symptoms to COVID-19 and therefore defer testing for enteric 
Fig. 1 Trends in stool testing and results over time during COVID-19 pandemic $(2 / 1 / 2020-4 / 11 / 2020)$ for a $C$. difficile testing and $\mathbf{b}$ gastrointestinal PCR testing
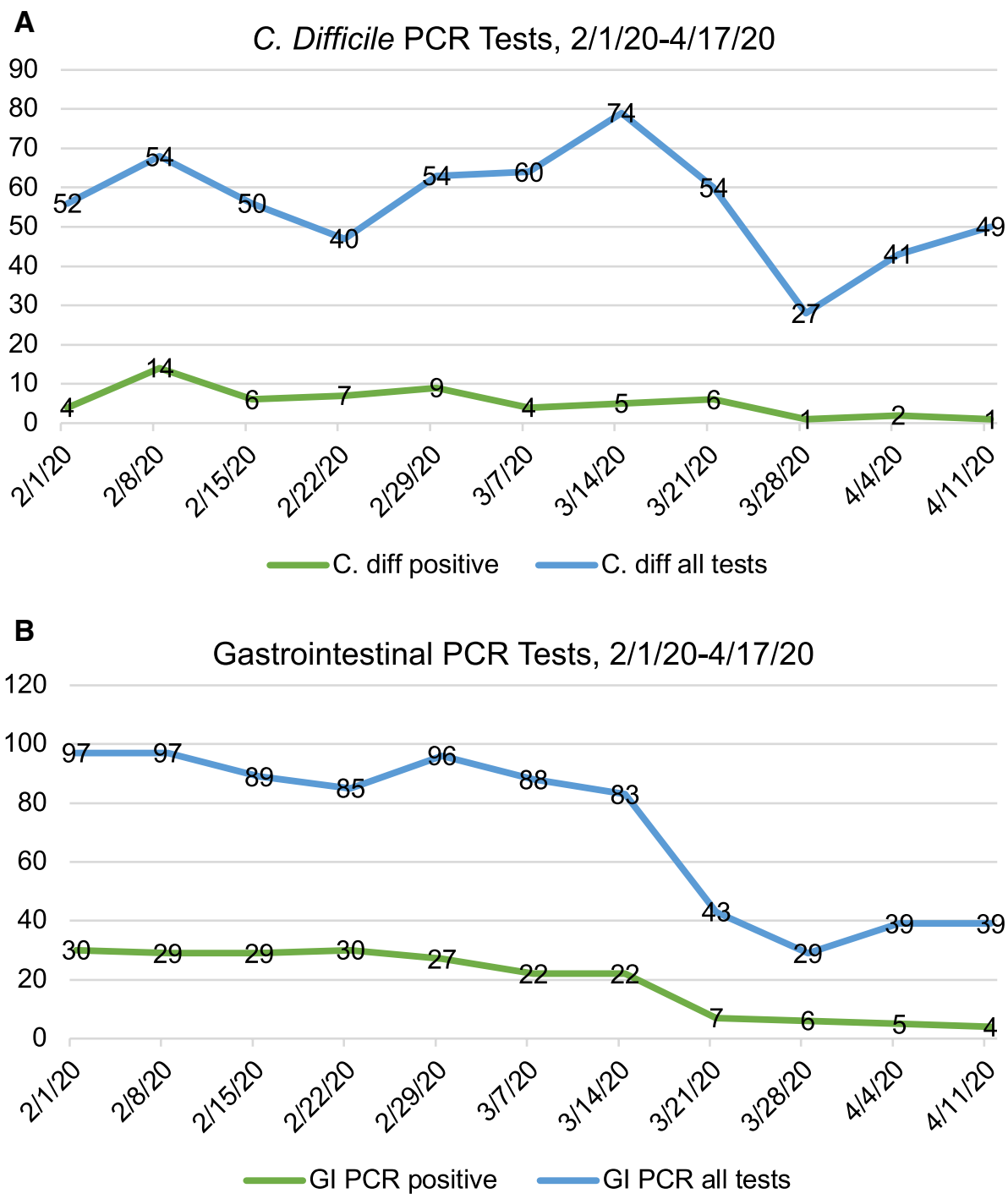

pathogens (although testing rates were significantly higher in COVID-19 positive than negative patients). It is also possible that, due to concerns over fecal shedding [17], providers may have been discouraged from ordering stool tests to limit nursing contact with stool specimens during collection. Not surprisingly, the rate of $C$. difficile testing, which nadired on March 28, seemed to subsequently be increasing. This is expected based on the number of critically ill patients and number of patients requiring broad spectrum antibiotics and longer hospital length of stay as the COVID-19 pandemic continued.

There are limitations to this study inherent to its retrospective and observational nature. These associations do not establish causality, and there may be an inherent bias in the types of patients that providers chose to test with stool studies in the setting of the pandemic. While this study was performed in high-volume centers, the testing practice may be subject to institutional biases limiting generalizability. The ordering of the tests was done at the discretion of the physician caring for the patient. For some patients, only one of the two stool tests were available. There may have been differences in the approach to the workup of diarrhea given physicians were redeployed from different specialties and different institutions. Some may not have been aware of the availability of GI PCR testing options. Since the tests are collected in distinct containers, it is possible some may not have been run due to improper collection. Furthermore, both tests may not have been appropriate depending on the clinical scenario. We included all available results to maximize the available data. This study highlights the need for ongoing awareness among clinicians regarding the correct diagnosis of GI infections, particularly C. difficile, in these populations.

Despite the large study size, a relatively small absolute number of patients $(311,6.3 \%)$ underwent stool tests. 
Prior reports from our institution demonstrated that $18 \%$ of individuals tested for COVID-19 presented with diarrhea, and $35 \%$ presented with gastrointestinal symptoms including nausea and vomiting [3]. Given that stool testing decreased institutionally as diarrhea become known as a COVID-19-related symptom, the actual prevalence of enteric infection among patients may have been underestimated in our study. In addition, a positive result on a gastrointestinal PCR test could represent chronic shedding or potential false positive, in addition to actual infectious gastroenteritis.

\section{Conclusions}

In conclusion, we identified that COVID-19 positive patients were less likely to test positive for enteric infections than COVID-19 negative patients overall; however, the frequency of $C$. difficile, in particular, was not significantly different between the two groups. Given the high prevalence of GI symptoms in COVID-19 patients, there may be decreased utility in using extensive gastrointestinal pathogen panels, as pathogens aside from $C$. difficile rarely contribute to diarrhea in COVID-19 positive patients. Our results suggest the need for ongoing vigilance regarding $C$. difficile infection in these populations. Furthermore, the possible long-term impact of COVID-19 on gastrointestinal problems as well as risk for future infections requires further study.

\section{Acknowledgment None.}

Author's contribution ML, JK, ASF, AMJ, CH, and DEF contributed to study concept and design. ML, JK, ASF, MI, HT, ERS, and BM contributed to acquisition of data. ML, JK, ASF, MI, HT, ERS, BM, WGG, JZ, BL, CH, and DEF contributed to analysis and interpretation of data. ML, JK, and AMJ contributed to drafting of the manuscript. ML, JK, ASF, AMJ, HT, ERS, MI, BM, WGG, JZ, BL, DEF, and CH contributed to critical revision of the manuscript for important intellectual content. All authors approved the final version of the manuscript.

Funding ASF: NIH T32DK083256, BL: The Louis and Gloria Flanzer Philanthropic Trust.

\section{Compliance with Ethical Standards}

Conflict of interest They authors declare that they have no conflict of interest.

\section{References}

1. Huang C, Wang Y, Li X et al. Clinical features of patients infected with 2019 novel coronavirus in Wuhan. China. Lancet. 2020;395:497-506
2. Guan WJ, Ni ZY, Hu Y et al. Clinical characteristics of coronavirus disease 2019 in China. N Engl J Med. 2020;382:1708-1720

3. Nobel YR, Phipps M, Zucker J et al. Gastrointestinal symptoms and COVID-19: case-control study from the United States. Gastroenterology. 2020;159:e2

4. Redd WD, Zhou JC, Hathorn KE, McCarty TR, Bazarbashi AN, Thompson CC, Shen L, Chan WW. Prevalence and characteristics of gastrointestinal symptoms in patients with SARS-CoV-2 infection in the United States: A multicenter cohort study. Gastroenterology. 2020

5. Hajifathalian K, Krisko T, Mehta A, et al. Gastrointestinal and hepatic manifestations of 2019 novel coronavirus disease in a large cohort of infected patients from New York: clinical implications [published online ahead of print, 2020 May 8]. Gastroenterology. 2020; https://doi.org/10.1053/j.gastro.2020.05.010

6. Cholankeril G, Podboy A, Aivaliotis VI, Tarlow B, Pham EA, Spencer S, Kim D,Hsing A, Ahmed A. High prevalence of concurrent gastrointestinal manifestations in patients with SARS-CoV-2: early experience from california. Gastroenterology. 2020

7. Pan L, Mu M, Yang P et al. Clinical characteristics of COVID19 patients with digestive symptoms in Hubei, China: a descriptive, cross-sectional, multicenter study. Am J Gastroenterol. 2020;115:766-773

8. Jin X, Lian JS, Hu JH et al. Epidemiological, clinical and virological characteristics of 74 cases of coronavirus-infected disease 2019 (COVID-19) with gastrointestinal symptoms. Gut. 2020;69:1002-1009

9. Zhou P, Yang XL, Wang XG et al. A pneumonia outbreak associated with a new coronavirus of probable bat origin. Nature. 2020;579:270-273

10. Zhang H, Kang Z, Gong H, et al. Digestive system is a potential route of COVID-19: an analysis of single-cell coexpression pattern of key proteins in viral entry process Gut 2020;69:1010-1018

11. Buss SN, Leber A, Chapin $\mathrm{K}$ et al. Multicenter evaluation of the BioFire FilmArray gastrointestinal panel for etiologic diagnosis of infectious gastroenteritis. J Clin Microbiol. 2015;53:915-925

12. Bélanger SD, Boissinot $\mathrm{M}$, Clairoux $\mathrm{N}$ et al. Rapid detection of clostridium difficile in feces by real-time PCR. J Clin Microbiol. 2003;41:730-734

13. Surawicz CM, Brandt LJ, Binion DG et al. Guidelines for diagnosis, treatment, and prevention of clostridium difficile infections. Am J Gastroenterol. 2013;108:478-498

14. Feuerstadt P, Das R, Brandt LJ. The evolution of urban C. difficile infection (CDI) in 2009-2011 is less severe and has better outcomes than CDI in 2006-2008. Am J Gastroenterol 2014; $109: 1265-1276$

15. Han C, Duan C, Zhang S, et al. Digestive symptoms in COVID19 patients with mild disease severity: clinical presentation, stool viral RNA testing, and outcomes. Am J Gastroenterol 2020:https ://doi.org/10.14309/ajg.0000000000000664.

16. Axelrad JE, Joelson A, Green PHR, Lawlor G, Lichtiger S, Cadwell K, Lebwohl B. Enteric infections are common in patients with flares of inflammatory bowel disease. Am J Gastroenterol. 2018;113:1530-1539

17. Chen $Y$, Chen L, Deng $Q$ et al. The presence of SARS-CoV-2 RNA in the feces of COVID-19 patients. J Med Virol. 2020;92:833-840

Publisher's Note Springer Nature remains neutral with regard to jurisdictional claims in published maps and institutional affiliations. 